\title{
Real Time Assistance for Stent Positioning and Assessment by Self-initialized Tracking
}

\author{
Terrence Chen ${ }^{1}$, Yu Wang ${ }^{3, \star}$, Peter Durlak ${ }^{2}$, and Dorin Comaniciu ${ }^{1}$ \\ ${ }^{1}$ Siemens Corporation, Corporate Research \& Technology, Princeton, NJ, USA \\ 2 Siemens Healthcare, Forchheim, Germany \\ ${ }^{3}$ Riverain Technologies, Miamisburg, OH, USA
}

\begin{abstract}
Detailed visualization of stents during their positioning and deployment is critical for the success of an interventional procedure. This paper presents a novel method that relies on balloon markers to enable real-time enhanced visualization and assessment of the stent positioning and expansion, together with the blood flow over the lesion area. The key novelty is an automatic tracking framework that includes a self-initialization phase based on the Viterbi algorithm and an online tracking phase implementing the Bayesian fusion of multiple cues. The resulting motion compensation stabilizes the image of the stent and by compounding multiple frames we obtain a much better stent contrast. Robust results are obtained from more than 350 clinical data sets.
\end{abstract}

Keywords: percutaneous coronary interventions, stent positioning, stent enhancement.

\section{Introduction}

Stent thrombosis and restenosis are associated with stent under-expansion, which has been shown as a major risk factor for patients undergoing percutaneous coronary intervention (PCI) 4]. This procedure is monitored by X-ray fluoroscopy where the guidewire, markers, or stent visibility are often quite low. Two of the major risk factors concern with the success rate of this procedure are: 1 . Whether the stent is implanted precisely at the desired location. 2. Whether the stent is expanded properly against the vessel wall. Due to poor contrast, clinical preference, and rapid motion of the coronaries, physicians often have difficulty making a precise judgement from live fluoroscopic images.

To overcome the problem where the low visibility of stent undermines the assessment of the stent implantation outcome, image processing techniques have been proposed to improve the image quality for better stent visibility. Most of the algorithms applied a motion-compensated noise reduction via landmark-based registration of multiple images [136]. Interested readers may refer to [2] for a comprehensive study of existing work.

\footnotetext{
* Contributions from Yu Wang are results from his internship at Siemens Corporation, Corporate Research and Technology.
} 
Existing solutions are mostly offline where the physician can only observe the enhanced stent from one static image. We propose a method to assist the physician in visualizing the stent during live monitoring. It is achieved by robust tracking of the balloon marker pair in an online manner such that real time guidance can be provided throughout the procedure. Physician can directly assess the stent expansion and the blood flow over the lesion area after stent implantation. It can also be applied to facilitating stent positioning. To achieve this goal, a robust self-initialized tracking framework is proposed. It can be directly applied to automation of other device tracking problems and potentially makes the image-based tracking techniques more practical in clinical applications.

Our work is essentially different from existing industrial solutions. Comparing to StentViz 11, Stentboost 9], ClearStent [8], and StentOptimizer [10, which apply post-processing to a short fluoroscopic acquisition to present an image of enhanced stent in relation to the vessel, our work has the following advantages: 1. Our work presents the enhanced stent in real time during acquisition. 2. The enhancement is visualized in every frame. On the other hand, comparing to Sync-RX [12], which also provides real-time solutions, our work has the following advantages: 1 . We propose a global optimization framework to use a few frames in the beginning for self-initialization. This improves the robustness of the tracking significantly (about 15\%). 2. The quality of our real time stent enhancement is much higher even for cases with low-dose radiation since we do not achieve the enhancement only by anchoring the original scene with the balloon marker locations. At last, our work provides one unique advantage, which is to allow the physician visualize the stent clearly in its original context. No information is lost or distorted in the whole scene. To the best of our knowledge, this is the first work to provide a clear stent in its original context. Our work is validated through a quantitative evaluation over more than $350+$ clinical scenes, a scale which is previously unreported.

\section{The Self-initialization Tracking Framework}

Our tracking framework is illustrated in figure 11 (A), which consists of three phases - the self-initialization phase (I), the computation phase (II), and the online tracking phase (III). For a specific tracking target (in our applications, the balloon marker pair), we assume an offline detector $\mathbf{D}$ is trained. It can be applied to an image and output multiple candidates of the target object with confidence scores. In our work, a probabilistic boosting tree [7] is used as the classifier to train the offline detector $\mathbf{D}$. In phase I, we collect $n$ frames $(n=10$ by default), apply $\mathbf{D}$ to all images, and then localize the target pattern, balloon marker pair, in every frame by the spatial-temporal cues. A few frames (4 to 5 frames in our cases) may be skipped during computation of phase I, which is named the computation phase (II) in this paper. Once the computation is done, real time tracking can be performed to track the target pattern online. 


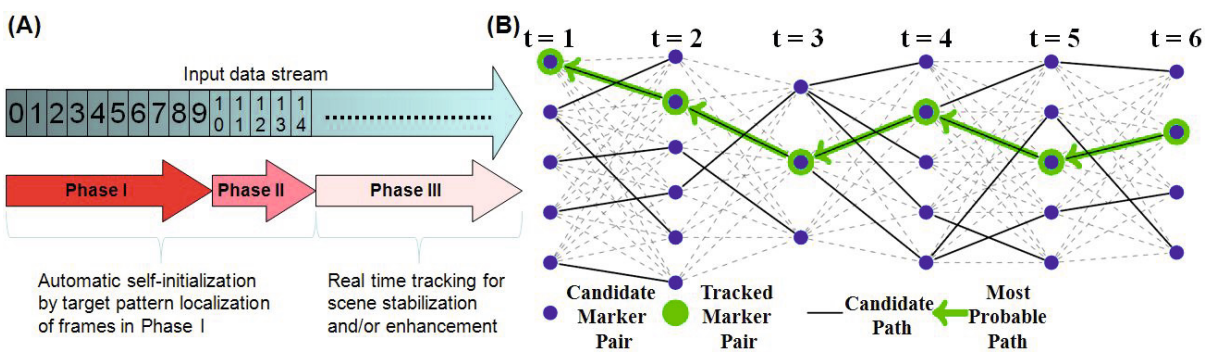

Fig. 1. (A) The proposed tracking framework (B) The trellis graph of the Viterbi algorithm. Each node represents a marker pair. The bold green path is the most probable path/viterbi path.

\subsection{Automatic Self-initialization}

To automatically initialize the tracking process, we take the first $n$ frames $(n=$ 10) from the live fluoroscopic stream and apply a novel offline tracking method based on the Viterbi algorithm to find the marker pair sequence in a globally optimal manner. In addition, results in phase I are used to create an adaptive joint marker pair template $\mathbf{T}$ and statistical models that can robustify the online tracking phase. Specifically, after obtaining detection results by applying $\mathbf{D}$ to the first 10 frames, the goal is to find the best sequence of the marker pairs that maximizes the following posterior probability:

$$
\hat{X}=\arg \max _{X} P(X \mid \mathbf{Y}),
$$

where $X=\left\{x_{1}, \ldots, x_{T=10}\right\}$ is the state sequence, and $x_{t, k}=\left(b_{t, k}, e_{t, k}\right)$ is the $k$ th state of the marker pair $b_{t, k}$ and $e_{t, k}$ at time $t . \mathbf{Y}=\left\{I_{1}, \ldots, I_{T=10}\right\}$ are the images. This problem is solved by the Viterbi algorithm which recursively finds the weight of the most likely state sequence ending with each $x_{t, k}\left(V_{t, k}\right)$ at $t$ :

$$
\begin{aligned}
& V_{1, k}=P\left(I_{1} \mid x_{1, k}\right) P\left(x_{1, k}\right) ; \\
& V_{t, k}=P\left(I_{t} \mid x_{t, k}\right) \max _{j}\left(P\left(x_{t, k} \mid x_{t-1, j}\right) V_{t-1, j}\right),
\end{aligned}
$$

where, $P\left(x_{1, k}\right)$ represents the prior probability of the $k$ th marker pair at the first frame and is set to $1 . P\left(I_{t} \mid x_{t, k}\right)$ represents the observation probability defined as the sum of the detection confidences of both markers:

$$
P\left(I_{t} \mid x_{t, k}\right) \propto P\left(I_{t} \mid b_{t, k}\right)+P\left(I_{t} \mid e_{t, k}\right)
$$

$P\left(x_{t, k} \mid x_{t-1, j}\right)$ represents the transition probability from the $j$ th state of time $t-1$ to the $k$ th state of time $t$ and is defined as a combination of length and direction consistencies, and the movement probability:

$$
P\left(x_{t, k} \mid x_{t-1, j}\right) \propto U_{\text {motion }} * U_{\text {length }} * U_{\text {dir }},
$$


where $U_{\text {motion }}=\exp \left(-\left(\left\|b_{t, k}-b_{t-1, j}\right\|+\left\|e_{t, k}-e_{t-1, j}\right\|\right) / \sigma\right)$ and the $\sigma$ is set to 100 in our experimental studies, $U_{\text {length }}=\exp \left(-\left|l_{t, k}-l_{t-1, j}\right| / l_{t-1, j}\right)$, and $U_{\text {dir }}=\max \left(\left(b_{t, k}-e_{t, k}\right) \cdot\left(b_{t-1, j}-e_{t-1, j}\right) /\left(l_{t, k} \cdot l_{t-1, j}\right), 0\right)$ with $l_{t, k}=\left\|b_{t, k}-e_{t, k}\right\|$ and $l_{t-1, j}=\left\|b_{t-1, j}-e_{t-1, j}\right\|$. The most probable path found by the Viterbi algorithm is illustrated in Figure 1 (B). The best state in time $T$ is found as $\hat{x}_{T}=\arg \max _{x_{T, k}}\left(V_{T, k}\right)$, and the states in other time slices are recovered by backtracking the Viterbi path. Once the best path is found, a simple heuristic is applied to invalidate frames with inconsistent motion or marker pair distance.

\subsection{Online Tracking}

Tracking by Detection. In the online tracking phase, $\mathbf{D}$ is also applied to generate marker pair candidates with confidence scores at each frame. A Bayesian inferencing framework to maximize the posterior probability of the balloon marker pair model $x_{t}$ at time $t$ is formulated as:

$$
\hat{x}_{t}=\arg \max _{x_{t}} P\left(x_{t} \mid I_{1 \ldots t}\right),
$$

where $I_{1 \ldots t}$ is image observation from 1 to $t$-th frame. By assuming a Markovian representation of the stent motion the above formula can be expanded as:

$$
\hat{x}_{t}=\arg \max _{x_{t}} P\left(I_{t} \mid x_{t}\right) \int_{x_{t-1}} P\left(x_{t} \mid x_{t-1}\right) P\left(x_{t-1} \mid I_{1 \ldots t-1}\right) d x_{t-1} .
$$

Eq. (7) essentially combines two parts: the likelihood term, $P\left(I_{t} \mid x_{t}\right)$, and the prediction term, $P\left(x_{t} \mid x_{t-1}\right)$. The likelihood term $P\left(I_{t} \mid x_{t}\right)$ is estimated by combining the detection score from $\mathbf{D}$ and the template matching as follows:

$$
P\left(I_{t} \mid x_{t}\right)=P\left(Z_{t} \mid x_{t}\right) \cdot P\left(C_{t} \mid x_{t}\right)
$$

where $P\left(Z_{t} \mid x_{t}\right)$ is probability measure of the marker pair at the $t$-th frame and is obtained directly by D. $P\left(C_{t} \mid x_{t}\right)$ is obtained by the cross-correlation between a local patch defined by the current evaluating marker pair at frame $t$ and the same-sized patch defined by the marker pair in the adaptive joint template $\mathbf{T}$ generated from the initialization phase.

The motion smoothness term $P\left(x_{t} \mid x_{t-1}\right)$ in (7) is formulated as a combination of translation, orientation, and shape consistencies of the joint marker pair at time $t$ compared to the joint marker pair at time $t-1$. The translation and shape consistencies are defined based on the Gaussian models $\left(l \sim \mathcal{N}\left(u_{\text {length }}, \sigma_{\text {length }}\right)\right.$, $\left.\left.w \sim \mathcal{N}\left(u_{\text {width }}, \sigma_{\text {width }}\right), h \sim \mathcal{N}\left(u_{\text {height }}, \sigma_{\text {height }}\right), m \sim \mathcal{N}\left(u_{\text {motion }}, \sigma_{\text {motion }}\right)\right)\right)$ generated in initialization phase to model the lengths, widths and heights (the bounding box of the marker pair), and movements of the marker pair, respectively. In the online tracking phase, the parameters of the marker pair length model are updated with the length of the tracked marker pair $\left(\hat{l}_{t}\right)$ as follows: 


$$
\begin{aligned}
u_{\text {length }}^{t} & =(1-\lambda) u_{\text {length }}^{t-1}+\lambda \hat{l}_{t} \\
\left(\sigma_{\text {length }}^{t}\right)^{2} & =(1-\lambda)\left(\sigma_{\text {length }}^{t-1}\right)^{2}+\lambda\left(\hat{l}_{t}-u_{\text {length }}^{t}\right)^{2}
\end{aligned}
$$

where $\lambda$ is the learning rate and is set as 0.1 in our studies. The other Gaussian models are updated in the same manner in the online tracking phase.

The joint template $\mathbf{T}$ is also updated online during the online tracking phase with new template $\mathbf{T}_{t}$ using the following equation:

$$
\mathbf{T}=(1-\lambda) \mathbf{T}+\lambda \mathbf{T}_{t},
$$

To reduce the false detection, top 5 candidates are sorted based on their scores and fed into a tracking validation process. The first valid marker pair is output as the final result. Our approach of validating the maker pair candidate online is based on the Gaussian models of lengths $\left(\mathcal{N}\left(u_{\text {length }}, \sigma_{\text {length }}\right)\right)$ and movements $\left(\mathcal{N}\left(u_{\text {motion }}, \sigma_{\text {motion }}\right)\right)$, a Gaussian model $\left(\mathcal{N}\left(u_{\text {tmp }}^{\text {joint }}, \sigma_{\text {tmp }}^{\text {joint }}\right)\right)$ of the matching scores (cross-correlation) between the joint template $\mathbf{T}$ and the template of one frame, and another Gaussian model $\left(\mathcal{N}\left(u_{t m p}^{p r e v}, \sigma_{t m p}^{p r e v}\right)\right)$ for the matching scores between templates at one frame and its previous frame. As a result, the marker pair is invalid if the length is abnormal (less than $u_{\text {length }}-\sigma_{\text {length }}$ or larger than $u_{\text {length }}+$ $\left.\sigma_{\text {length }}\right)$ or the motion is too large (larger than $\left.u_{\text {motion }}+\sigma_{\text {motion }}\right)$. The marker pair is also invalid if either one of the matching scores is too small (less than $u_{t m p}^{\text {joint }}$ $\sigma_{t m p}^{\text {joint }}$ or $\left.u_{t m p}^{\text {prev }}-\sigma_{t m p}^{\text {prev }}\right)$.

\section{Stent Positioning}

The first application we present using the proposed tracking framework is to facilitate stent positioning during PCI, where the main difficulty for physicians is to observe the balloon markers in relation with the lesion and vessels. This is sometimes quite challenging due to the rapid motion of coronaries during live monitoring. This procedure could be improved if a scene can be stabilized by the location of the balloon markers. For this purpose, we track the balloon marker pair in streaming data, transform the image such that the balloon marker pair is always aligned at exactly the same coordinates, and then display the transformed image to the physician. In our framework, $n=10$ is set in phase I. In a typical 15 fps acquisition rate, phase I takes 0.67 seconds, phase II takes 0.27 seconds, and followed by the real time online tracking. Once the online tracking starts, the new coming scene is stabilized by the coordinates of the balloon marker pair. Physician can inject contrast and observe the relation between the marker pair (undeployed stent) and the lesion area.

Real-time stent positining has been studied and is not a new application 12. Nevertheless, by the proposed framework of using a few frames for selfinitialization, it improves the robustness significantly. Applying a well-trained detector to any single image results in a detection accuracy of about $82 \%$ (c.f. Table 1]Part B. Accuracy of D). With the proposed framework, the initialization successful rate is validated to be improved to $97 \%$ (c.f. Table 1 the $3^{\text {rd }}$ row). 


\section{Stent Assessment}

In this section, we present a new way to assist physicians in assessing the stent expansion in real time by the same framework, which is the main application of the proposed paper. One additional step implemented here is that once the marker pairs are all localized in phase I, a motion-compensated based stent enhancement [6] is applied to obtain a stent enhancement image $(S E I)$. To make sure $S E I$ with good quality, $n=25$ is set in phase I. After the online tracking phase starts, a compound of $S E I$ and the streaming data is output as the visual result to the physician.

Specifically, let $f_{1}, \ldots, f_{t}$ be the $t$ frames of the input data stream, $S E I$ is obtained by $\left\{f_{1}, \ldots, f_{25}\right\}$. Once $S E I$ is obtained, a guidewire localization [6] technique is applied to $S E I$ to obtain the guidewire $\tau$ which passes through the two markers. A weighting field $\mathbf{W}$ is then generated by applying a Gaussian kernel to the guidewire location on SEI. Once phase III starts, which normally begins with $f_{40}$ to $f_{45}$, the $S E I$ is rigidly transformed to $S E I$ such that marker pair of $S E I$ is aligned with the marker pair in the current frame $f_{i}$. The output image $\hat{f}_{i}$ is then obtained by compounding the $S \hat{E} I$ and $f_{i}$ with $\mathbf{W}$ :

$$
\hat{f}_{i}=\mathbf{W} \times S \hat{E} I+(1-\mathbf{W}) \times f_{i},
$$

where $i$ is the frame index, which normally begins with 40 to 45 . In other words, the computation time for the $S E I$ is up to 3 seconds in a 15 fps acquisition. Figure 2 shows an example of the stent assessment results.

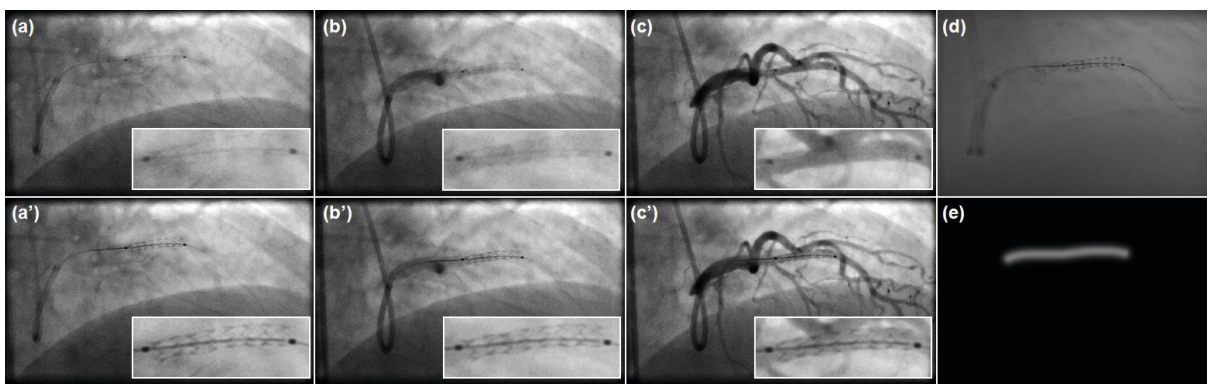

Fig. 2. $(a)(b)(c)$ : Three frames of the original scene; (a')(b')(c'): Corresponding frames of the output enhanced scene. Bottom right windows are zoom-in views of the stent; (d):Stent Enhancement Image (SEI) from the first 25 frames;(e): Weighting field (W)

Two notable comments we would like to address here are: 1 . If a live acquisition is followed by a playback, the same enhancement can be applied to frames in phase I and II as well. That is, during playback mode, stent is enhanced in every frame $\left(\left\{f_{1}, \ldots, f_{t}\right\}\right) 2$. Based on the clinical preference, during stent enhancement and assessment, scene stabilization based on the balloon markers can also be provided just like what we do during stent positioning. 


\section{Results}

356 clinical sequences with a total of 17234 frames are collected in PCI procedures from clinical sites in US, Europe, and Asia. They are either collected during stent positioning or after stent implantation, when the balloon is still in place. All the evaluated sequences have at least 20 frames, and 145 sequences have more than 50 frames. Image size ranges from $720 \times 720$ to $1024 \times 1024$. We evaluated the performance by a Intel Xeon PC $(2.2 \mathrm{GHz})$ in an online manner - the testing program sends one frame at a time by the speed of $15 \mathrm{fps}$. (Real time in this paper is defined as $15 \mathrm{fps}$ as this is the most common setting in real world clinics. Nevertheless, the online tracking of our algorithm performs far beyond this speed.) Both the results and processing time are monitored. In our evaluation, error is defined as the average displacement between the two markers to their ground truth positions. If the error is less than $1.5 \mathrm{~mm}$, it is regarded as correct. $1.5 \mathrm{~mm}$ is used to tolerate the manual annotation errors for small markers. The tracking accuracy after validation drops slightly from $98.5 \%$ to $97.7 \%$ if the error threshold is set as $0.6 \mathrm{~mm}$ (c.f. Table1]Part B. the last row).

We first compared the robustness of the proposed Viterbi algorithm for the self-initialization phase (I). Two measures are used for this evaluation - Success is defined by if all returned marker pairs are correct in phase I. Recall is defined by the number of marker pairs that are localized and returned in phase I. Table 1 Part A shows the results. $\mathbf{D}$ denotes the method if we directly apply $\mathbf{D}$ and use the top candidate as the marker pair. We also compared the method by Lu et al. [6]. It can be shown that the proposed method has the highest Success rate and can localize much more marker pairs than [6]. Please note that the evaluation were conducted for both stent positioning (POS) with 356 sequences and stent assessment (ASM) with 145 sequences.

Table 1. Part A. Evaluation and comparison for the self-initialization phase. Part B. Evaluation and comparison for phase III.

\begin{tabular}{|l|c|c||c|c|}
\hline Part A. & POS.Success & POS. Recall & ASM. Success & ASM. Recall \\
\hline \hline $\mathbf{D}$ & $0.68(285 / 356)$ & $100 \%$ & $0.52(75 / 145)$ & $100 \%$ \\
Lu et al. 6] & $0.96(342 / 356)$ & $78.9 \%$ & $0.95(138 / 145)$ & $79.2 \%$ \\
Proposed & $0.97(345 / 356)$ & $88.9 \%$ & $0.97(140 / 145)$ & $90.3 \%$ \\
\hline Part B. & Accuracy & Accuracy after validation & FPS \\
\hline \hline D & $81.93 \%(11203 / 13674)$ & \multicolumn{2}{|c|}{ N/A } & 35.54 \\
Online Boosting & $53.00 \%(7247 / 13674)$ & N/A & 9.08 \\
Proposed method & $96.50 \%(13195 / 13674)$ & $98.50 \%(12651 / 12844)$ & 29.04 \\
\hline
\end{tabular}

Next, we evaluated our tracking method in phase III. For this experiment, we also compared with the online boosting (OB) detector [5]. In the experiments with the online boosting detector, we initialized two online boosting detectors that track the markers independently. 50 base classifiers are used in each detector 
and the Haar features are employed. At each frame, the online boosting detector is updated with the tracking result $(20 \times 20$ window centering at the tracked marker) as the positive sample and randomly sampled windows in the neighboring region as the negative samples. We evaluated the tracking by number of correctly tracked frames. Since there are more frames (13674) used in the stent positioning procedure, the evaluation was conducted by this procedure only. For fair comparison, the same initialization was applied for all methods. As shown in Table 1 Part B, the proposed online tracking method outperformed the other two tacking methods and reached $96.5 \%$ accuracy and an average speed of 29.04 FPS. After applying the validation approach the accuracy is boosted to $98.5 \%$.

We report the running time of the entire workflow below. In the stent positioning procedure, the self-initialization of 10 frames takes an average of 0.27 seconds after phase I, which means 4 to 5 frames are needed for phase II. For the stent assessment procedure, the self-initialization of 25 frames takes an average of 0.36 seconds after phase I. The registration of all frames and generation of the $S E I$ takes about additional 0.82 seconds. In total, a 1.18 second is needed for phase II, which is about 15 to 20 frames. Online stent enhancement can start to be displayed after about 40 to 45 frames.

In summary, main advantages of the proposed tracking framework include: 1. It is more efficient and accurat than manual initialization, and it is more robust than a single frame initialization. 2. Results obtained at phase I can be used to learn the scene specific statistics and provide an appearance template to improve the tracking robustness in phase III. This is another key factor how we achieve such robust tracking performance.

\section{Conclusion}

We present a framework to assist stent positioning and post implantation assessment, which provides physicians a clearly observable stent in real time fluoroscopy. The proposed framework can be potentially applied to other interventional applications to streamline the workflow and improve the results.

\section{References}

1. Bismuth, V., Vaillant., R.: Elastic registration for stent enhancement in X-ray image sequences. ICIP (2008)

2. Bismuth, V., Vaillant., R., Funck, F., Guillard, N., Najman., L.: A comprehensive study of stent visualization enhancement in X-ray images by image processing means. In: Prince, J. L., Pham, D. L., Myers, K. J. (eds.) Medical Image Analysis, vol. 15, No. 4:565-76, Springer (2011)

3. Florent, R., Nosjean, L., Lelong, P., Rongen, P.M.J.: Medical viewing system and method for enhancing structures in noisy images. US Patent 7415169. 
4. Fujii, K., Carlier, S.G., Mintz, G.S., Yang, Y.M., Moussa, I., Weisz, G., Dangas, G., Mehran, R., Lansky, A.J., Kreps, E.M., Collins, M., Stone, G.W., Moses, J.W., Leon., M.B.: Stent underexpansion and residual reference segment stenosis are related to stent thrombosis after sirolimus-eluting stent implantation: An intravascular ultrasound study. Journal of the American College of Cardiology. 45:995-998 (2005)

5. Grabner, H., Bischof, H.: On-line Boosting and Vision. CVPR (2006)

6. Lu, X., Chen, T., Comaniciu, D.: Robust Discriminative Wire Structure Modeling with Application to Stent Enhancement in Fluoroscopy. CVPR (2011)

7. Tu, Z.: Probabilistic boosting-tree: Learning discriminative models for classification, recognition, and clustering. ICCV (2005)

8. ClearStent, http://www.swe.siemens.com/france/web/fr/med/produits/angio/ solutions/applications_cliniques/Pages/IC-stent.aspx

9. StentBoost, http://www.gehealthcare.com/euen/interventional_xray/ clinical_cases/bifurcation_lesion.html

10. StentOptimizer, http://www . paieon. com/Products . asp?Par=9.27\&id=75

11. StentViz, http://www.genewscenter.com/content/detail.aspx?ReleaseID= 8465\&NewsAreaID $=2$

12. Sync-RX, http://www. sync-rx.com/EN/contents/page.aspx?contentPageID=13 